\title{
No-reference Blur Estimation Based on the Average Cone Ratio in the Wavelet Domain
}

\author{
Ljiljana Platiša, Aleksandra Pižurica, Ewout Vansteenkiste, Wilfried Philips \\ Ghent University, Department of Telecommunications and Information Processing \\ TELIN-IPI-IBBT, St-Pietersnieuwstraat 41, B-9000 Ghent, Belgium
}

\begin{abstract}
We propose a wavelet based metric of blurriness in the digital images named CogACR Center of gravity of the Average Cone Ratio. The metric is highly robust to noise and able to distinguish between a great range of blurriness. To automate the CogACR estimation of blur in a no-reference scenario, we introduce a novel method for image classification based on edge content similarity. Our results indicate high accuracy of the $\operatorname{Cog} \mathrm{ACR}$ metric for a range of natural scene images distorted with the out-of-focus blur. Within the considered range of blur radius of 0 to 10 pixels, varied in steps of 0.25 pixels, the proposed metric estimates the blur radius with an absolute error of up to 1 pixel in 80 to $90 \%$ of the images.
\end{abstract}

